\title{
Intratunical injection of autologous adipose stromal vascular fraction reduces collagen III expression in a rat model of chronic penile fibrosis
}

\author{
Lukman Hakim $^{1,2} \cdot$ Salvatore Fiorenzo $^{3}$ - Petter Hedlund ${ }^{4,5} \cdot$ Francesco Montorsi $^{6}$ - Trinity J. Bivalacqua ${ }^{7}$. \\ Dirk De Ridder $^{1} \cdot$ Emmanuel Weyne $^{1} \cdot$ David Ralph $^{8} \cdot$ Giulio Garaffa $^{8} \cdot$ Asif Muneer $^{8,9}{ }^{8,9}$ Steven Joniau (iD) ${ }^{1}$. \\ Maarten Albersen ${ }^{1} \cdot$ Fabio Castiglione ${ }^{1,6,8} \cdot$ On behalf of the Trauma and Reconstructive Urology Working \\ Party of the European Association of Urology (EAU) Young Academic Urologists (YAU)
}

Received: 1 November 2018 / Revised: 27 January 2019 / Accepted: 8 March 2019

(c) Springer Nature Limited 2019

\begin{abstract}
Previous studies have shown that the injection of adipose stem cells and stromal vascular fraction(SVF) into the tunica albuginea (TA) during the inflammatory phase in a rat model of Peyronie's disease(PD) prevented the development of TA fibrosis. Our aim was to investigate whether local injection of SVF can reduce established fibrosis in a rat model of chronic phase of PD. Eighteen-male 12-wk-old Sprague-Dawley rats were divided in three equal groups: sham, PD without treatment (PD) and PD treated with SVF(PD-SVF). Sham rats underwent 2 injections of vehicle into the TA one month apart. PD rats underwent TGF- $\beta 1$ injection and injection of vehicle one month later. PD-SVF rats underwent TGF- $\beta 1$ injection followed by SVF (1-million cells) one month later. One month after the last treatment, the animals, $n=6$ rats per group, underwent measurement of intracorporal and mean arterial pressure during electrostimulation of the cavernous nerve. Following euthanasia, penises were harvested for in-vitro study. Erectile function was not statistically significantly different between groups. PD animals developed subtunical areas of fibrosis and elastosis with upregulation of collagen III protein. These fibrotic changes were reversed after injection of SVF. We provide evidence that local injection of SVF reverses TA fibrosis in a rat model of chronic phase of PD.
\end{abstract}

\section{Introduction}

Fibrosis is defined by an excessive accumulation of extracellular connective tissue proteins (extracellular matrix (ECM)) such as collagen, elastin and fibronectin [1]. Typically, ECM aggregation is an indispensable and reversible

Maarten Albersen

maartenalbersen@hotmail.com

1 Laboratory for Experimental Urology, Organ systems, Department of Development and Regeneration, University of Leuven, Leuven, Belgium

2 Department of Urology, Airlangga University / Dr Soetomo General Hospital, Surabaya, Indonesia

3 Facoltà di Medicina e Chirurgia, Unversitá Degli Studi di Palermo, Palermo, Italy

4 Department of Clinical and Experimental Pharmacology, Lund University, Lund, Sweden phase of the wound healing process [2]. It can, however, progress into long-lasting fibrotic response if the woundhealing process itself becomes deregulated [3]. Fibrosis represents the final, usual pathological result of many chronic inflammatory conditions $[1,4]$. Peyronie's Disease (PD) is an acquired fibrotic disorder involving the tunica albuginea (TA) 
of the penis and leading to the formation of fibrous plaques, penile curvature, pain and rarely erectile dysfunction [5].

Commonly, PD is classified into an acute (or inflammatory) phase and a chronic (or stable) phase. During the former, there may be penile pain and a penile deformity may become visible during erection. Penile pain resolves spontaneously within 12-18 months of PD onset in most patients [5]. During the chronic phase pain is absent and the curvature is stabilized [6].

In the last decade, several preclinical studies have suggested a possible role of adipose stem cell (ADSC) in preventing penile tunica albuginea fibrosis in a rat model of acute PD [1]. On the other hand there is a void in the existing literature on whether ADSC treatment is efficacious in reversing fibrosis once a plaque has been established [7]. This aspect is of utmost importance for the translational point of view as the majority of patients presents with an established plaque [5]. In addition, it is worth to remember that there are several issues tied to the use of culture-expanded ADSC such as potential contamination, genetic instability and oncogenesis [8].

Adipose SVF is isolated as part of the aqueous fraction derived from enzymatic digestion of fat tissue [9]. SVF is a heterogeneous cell fraction including endothelial cells, smooth muscle cells, macrophages, regulatory T-cells, fibroblasts, and a large population of stem cells [10]. Several studies have explored the efficacy of adipose SVF in fibrotic diseases with positive outcome [11-13]. Interestingly, in animal models of erectile dysfunction [14] and incontinence [15], SVF treatment showed positive results and efficacy comparable to culture-expanded ADSC. More important, unlike ADSC, SVF is autologous, much more easily acquired, without the need for any cell separation or culturing conditions, and can be performed within a $1.5 \mathrm{~h}$ time-frame in a closed system thus limiting the risk of contamination and making it relatively safe [16].

The rat is most commonly animal model for PD due to the analogous morphological and biological penile characteristics with humans. In addition, this animal model presents with low costs for purchase and maintenance. More important, Bivalacqua et al. showed that injection of the TGFb1 in penile rat induced PD-like changes including erectile dysfunction [17].

In our recent study we showed that SVF are able to prevent TA fibrosis formation in a rat model of early phase of PD [18]. In the current study, we investigated the antifibrotic effects of a local injection of SVF after establishment of TA fibrosis in a validated rat PD model.

\section{Methods}

\section{Ethical approval}

All experiments on animal tissues were approved by the ethics committee of University of Leuven (P 272/2014).
We calculated a sample size of 18 considering 3 groups (6 animals for each group), a statistical power of 0.9 , effect size d: 2, alpha level $0.05\left(\mathrm{G}^{*}\right.$ Power 3.1).

\section{Animals}

Male Sprague-Dawley rats $(n=18 ; 12$ wk. old; 300-350 g; Charles River Laboratories, Wilmington, MA, USA) were used. Rats were housed in pairs under 12-h reversed cycle lighting with ad libitum access to food and water. Intraperitoneal ketamine $(75 \mathrm{mg} / \mathrm{kg})$ and xylazine $(50$ $\mathrm{mg} / \mathrm{kg}$ ) were used for anaesthesia for the surgical procedure. Amoxicillin $(50 \mathrm{mg} / \mathrm{kg}$ intraperitoneally) was administered $1 \mathrm{~h}$ prior to the surgical procedures as prophylaxis. Rats were euthanized using carbon dioxide asphyxia.

\section{Isolation of SVF}

Animals underwent resection of the para-testicular adipose tissue through abdominal midline incision as previously described [19]. The adipose tissue was rinsed with PBS, minced into small pieces, and then incubated in a solution containing $0.075 \%$ collagenase type IA (Sigma-Aldrich, St. Louis, MO, USA) for $1 \mathrm{~h}$ at $37^{\circ} \mathrm{C}$ with vigorous shake for $15 \mathrm{~s}$ in 20 -min intervals. The top lipid layer was removed, and the remaining liquid portion was centrifuged at $1000 \times g$ for $10 \mathrm{~min}$ at room temperature. The cells were then washed, centrifuged, and the pellet was resuspended in saline. The nucleated cells from the pellet were counted and diluted to 5000 cells per $\mu \mathrm{l}$ in saline, of which $200 \mu \mathrm{l}$ were kept on ice until injection [19].

\section{Study design}

Rats were not-randomly divided into 3 equal groups. The sham group $(n=6)$ underwent injection of $50-\mu 1$ vehicle (citrate buffer) in the dorsomedial aspect of the right midshaft $\mathrm{TA}$ with a microliter syringe after opening the Buck's fascia as previously described. The remaining 12 animals were injected with recombinant $0.5 \mu \mathrm{g}$ of transforming growth factor (TGF)- $\beta 1$ in $50-\mu \mathrm{l}$ vehicle [20]. After 1 month, all rats underwent to resection of the para-testicular adipose tissue and received a second identical TA injection with either phosphate buffered saline (PBS; sham and PD group) or autologous SVF in PBS (1 million cells in $200 \mu \mathrm{l}$ ) (PD-SVF group). Four weeks after the second treatment, 6 rats per group underwent in vivo erectile function evaluation after which the animals were euthanized, and the penises harvested for histological analysis and for protein extraction. 


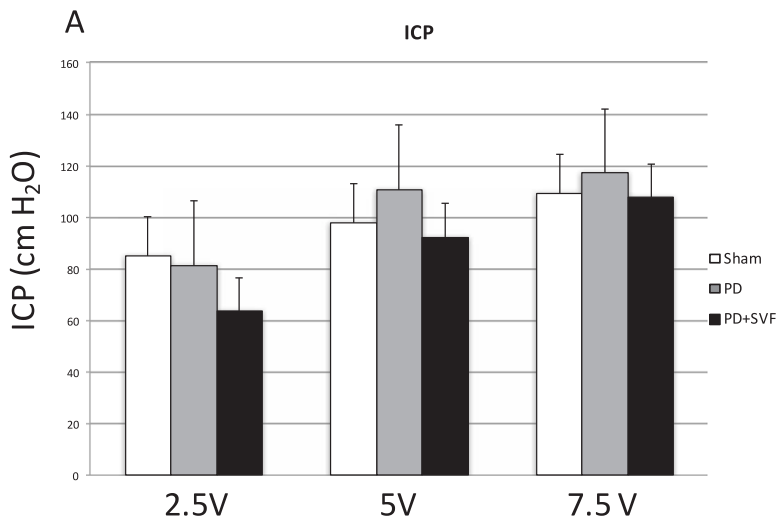

Fig. 1 Erectile function measurement: Summarized data comparing erectile function measurements in sham PD rats and rats treated with PD-SVF at various voltages during cavernous nerve electrostimulation. a Intracavernous pressure (ICP). b ICP normalized over mean

\section{Erectile function measurement}

Intracavernous pressure (ICP) response to electrostimulation of the cavernous nerve $(\mathrm{CN})$ was used to evaluate erectile function [20]. Briefly, under anaesthesia, the right $\mathrm{CN}$ was exposed and the right crus of the corpus cavernosum was identified and cannulated with a heparinized (200 $\mathrm{U} / \mathrm{ml}$ ) 25-G needle connected to a pressure transducer. The $\mathrm{CN}$ was activated $(2.5,5$, and $7.5 \mathrm{~V})$ by platinum electrodes connected to a stimulator at $20 \mathrm{~Hz}$ for $60 \mathrm{~s}$. The nerve was stimulated once per voltage, and a resting period of $2 \mathrm{~min}$ was allowed for nerve recovery between stimulations. Mean arterial pressure (MAP) was recorded by carotid artery cannulation.

\section{Histological analysis of tissue}

The penile midshaft at the level of the injection site was harvested, fixed, and further processed for histology. Haematoxylin and eosin and Masson's trichrome staining procedures were performed according to a standard protocol previously described [21].

\section{Western blot analysis}

Western blot was performed as previously described [2123] for the detection of collagen I, collagen III, and elastin proteins at the level of the penile midshaft. Glyceraldehyde-3-phosphate dehydrogenase or BetaActin were used as an internal standard. Primary antibodies were $\mathrm{Rb}$ Anti-Collagen III (Abcam Inc., Cambridge, MA, USA), Ms Anti-Elastin (1:500, Abcam Inc., Cambridge, MA, USA) and Rb controls against Beta-actin (B-actin) (1: 1000 Abcam Inc., Cambridge, MA, USA), Rb Anti-Collagen I (1:500; Abcam Inc., Cambridge, MA, USA) [22].

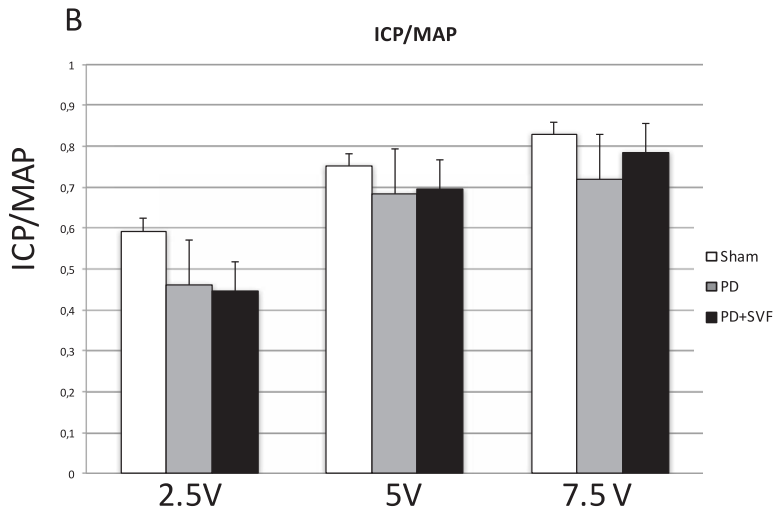

arterial pressure (MAP). $+\mathrm{p}<0.05$ versus both SHAM and PD-SVF in analysis of variance with post hoc Student-Newman-Keuls analysis

\section{Statistical analysis}

The results were analysed using Prism v.4 (GraphPad Software, San Diego, CA, USA) and expressed as mean standard deviation of the mean. Multiple groups were compared using one-way analysis of variance followed by the Student-Newman-Keuls test for posthoc comparisons. Statistical significance was set at $p<0.05$.

\section{Results}

No animals died during the experiment. No Major complication happened.

\section{Erectile function}

No significant difference was noted in ICP and ICP/MAP values between the three groups at $2.5,5$ and 7.5 volts $(p>$ 0.05 for all voltages) 4 weeks after vehicle or SVF injection (Fig. 1).

\section{Histological and western blot analysis}

Rats injected with TGF- $\beta 1$ (PD group) displayed a minimal deposition of amorphic matrix and a haphazard organization of collagen fibers in the TA which did not extend into the subtunical corpus cavernosum (Fig. 2). These morphologic results were corroborated by quantitative Western blot analysis, which revealed an increased protein content of collagen III and elastin compared to the sham group ( $p<0.05$ for both) (Fig. 3). In the SVF group, the overall structure of the TA and collagen III expression of the penile shafts were comparable to those of sham rats (Fig. 3). Collagen I/III ratio was higher in PD-SFV group comparing with the PD group ( $p$ : 0.02) (Fig. 3). Penile 


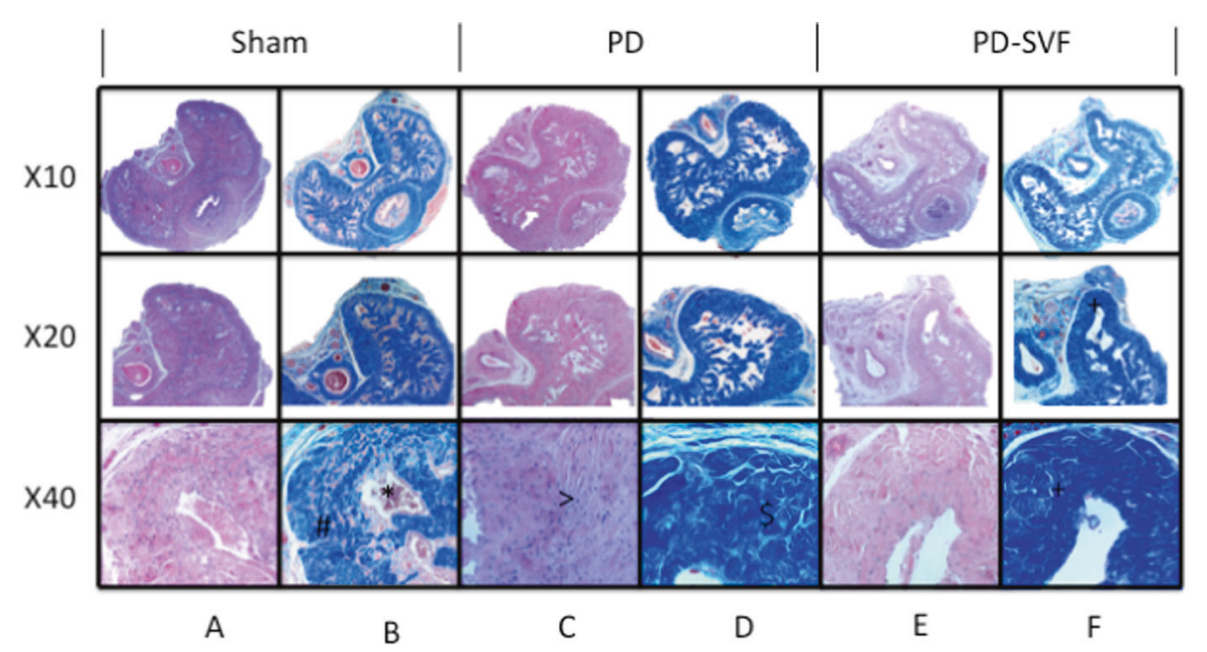

Fig. 2 Histology: Representative photomicrographs of Masson's trichrome and hematoxylin and eosin $(\mathrm{H} \& \mathrm{E})$ staining in midshaft sections of rat penises at magnification $\times 10, \times 20$, and $\times 40$. a $\mathrm{H} \& \mathrm{E}$ staining on sections from a Sham rat and (b) corresponding Masson's trichrome staining on an adjacent section from the same rat. $\mathbf{c} \mathrm{H} \& \mathrm{E}$ on sections from a PD rat and (d) corresponding. Masson's trichrome staining on an adjacent section from the same rat. e $\mathrm{H} \& \mathrm{E}$ sections from PD-SVF rat and (f) corresponding Masson's trichrome staining on an adjacent section from the same rat. Note the open cavernous

shafts from SVF-treated rats showed no significant differences in elastin expression compared to the sham and PD $(p>0.05)$. No significant differences in collagen I expression were noted between the three groups.

\section{Discussion}

The French Surgeon Francois de la Peyronie (1678-1747) first described PD describing the a series of patients with "rosary beads of scar tissue" [24]. PD is a relatively unknown condition, although recent studies suggest that it may occur in up to $9 \%$ of the male population [25]. Because of its impact on male sexual health, it can result in severe physical and psychological morbidity;[5] men may develop penile pain and curvature that may preclude sexual intercourse and reduce pleasure, with detrimental impacts on partner relationships. The pathology of the PD plaque has been investigated by several in vivo and in vitro studies. According to the available information, it is clear that fibrosis is the principal pathological component, combined with fibrin accumulation and different of inflammation degrees [1, 26-28].

The development of PD consists of an active and a chronic phase [5]. Compromised balance of the inflammatory factor production during the active phase causes abnormal wound healing, which in the chronic phase of PD results in collagen-elastin deposits and calcifications [1]. sinusoids in the sham rats $(*)$ and the surrounding normal bilayered structure of the tunica albuginea (\#). In PD rats, there is deposition of amorphic extracellular matrix material (\$) with scattered high numbers of cells $(>)$, which are expected to be fibroblasts based on their spindleshaped morphology and relationship with the extracellular matrix. In PD-SVF RAT, there is an increase in extracellular matrix deposition $(+)$; however, collagen fibers seem better organized and sinusoid structure is largely preserved

Fibrosis has been considered an inactive process, precluding organ regeneration $[1,29]$. However, in the last few decades, this perception has changed. Today, it is clear that fibrosis is not static nor irreversible, but instead the consequence of an incessant remodelling that makes it subject to therapeutic intervention [1]. The most important challenge in fibrosis is to halt fibrogenesis and reverse established fibrosis without delaying wound healing process. Consequently, our increased understanding of fibrosis, its dynamics, and the potential of fibrotic microenvironments to reverse holds promise for the development of highly specific antifibrotic therapies. Stem cells (SCs) have been well known for their ability to differentiate to several types of cell populations [30, 31]. However, this ability is not the only feature that makes these cells appealing for therapeutic application. The secretions of a broad range of paracrine factors by SCs includes growth factors, cytokines, chemokines and even functional small RNAs via extracellular vesicles, which allow these cells to influence and modify their host environment, especially during, and early after injury to the tissue $[1,30,31]$. In recognition of these unique properties, there is an increasing body of evidence for the role of SCs as potential treatment strategy to alleviate fibrosis [2, 4, 29, 32]. In particular, mesenchymal stem cells (MSC) and SVF have been a popular SC type in this context [1].

Although the exact MSC anti-fibrotic mechanisms remain to be clarified, the principal theory is that they act as 


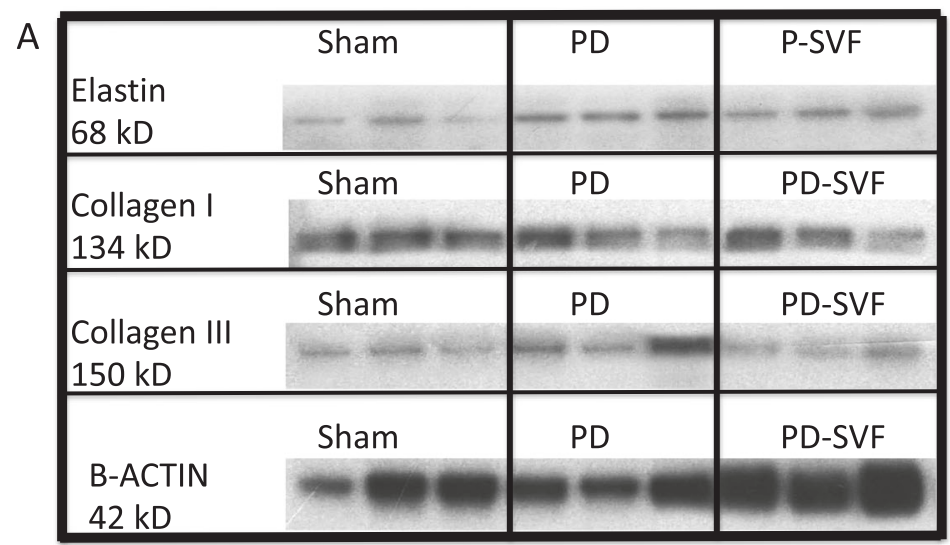

B

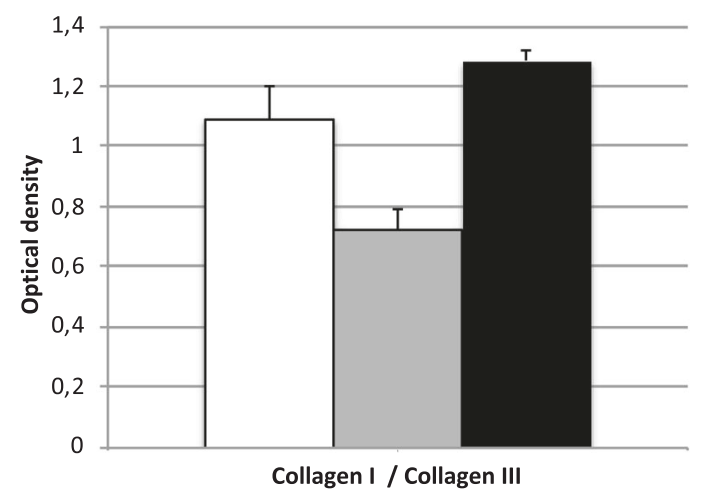

D

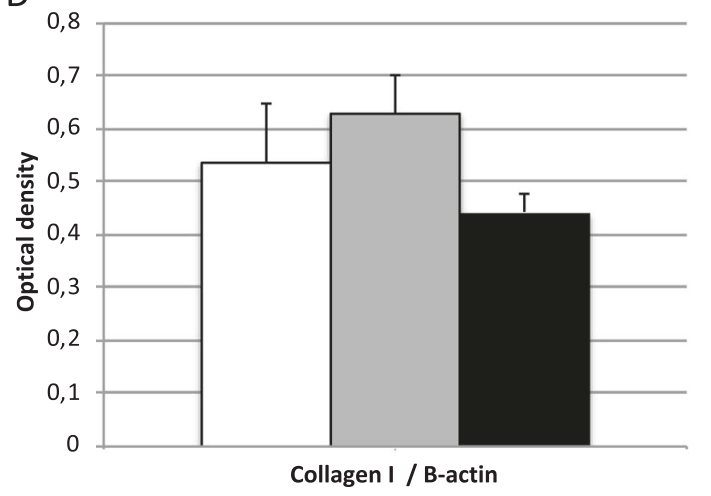

C

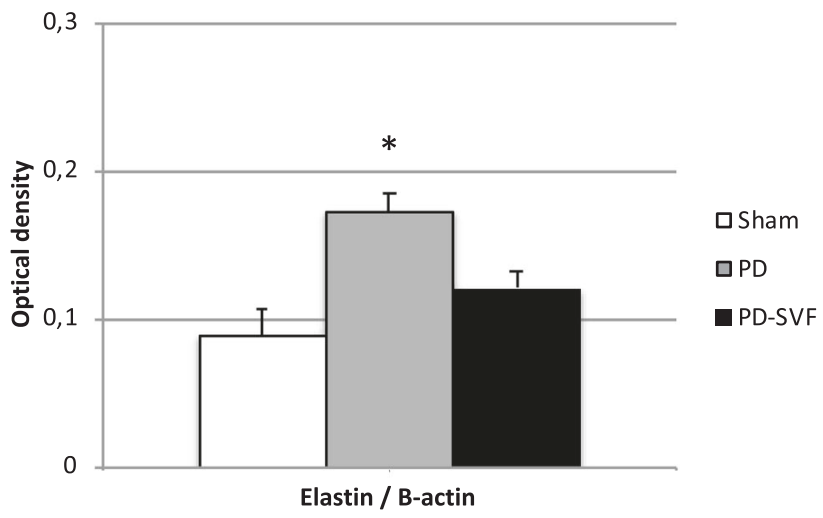

$\mathrm{E}$

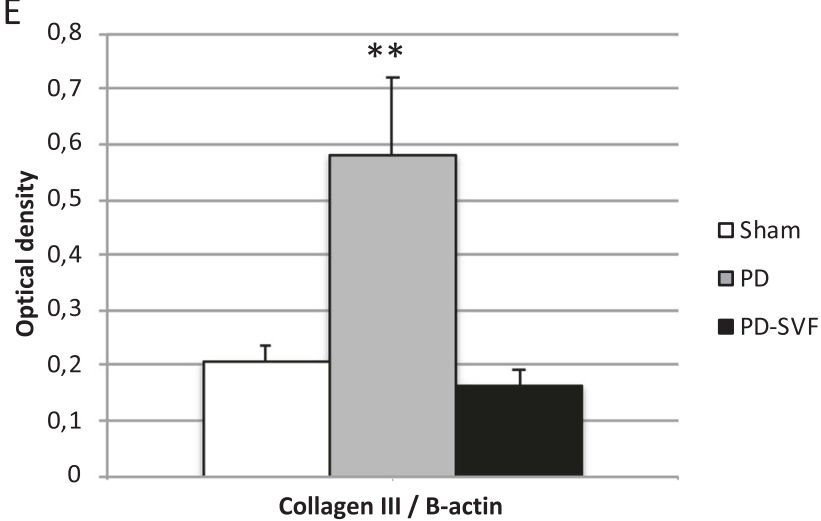

Fig. 3 Western blot analysis for collagen III, Collagen I and elastin. a Representative chemiluminescence images of blotted membranes containing protein extracts of all three groups. Double bands are due to binding of antibodies to glycosylated and nonglycosylated forms of these molecules. b Collagen I and III expression ratio $* * p<0.05$ versus both Sham and PD-SVF in analysis of variance with post hoc Student-Newman-Keuls analysis. c Summarized protein expression

a "drug-store", affecting several pro-fibrotic factors simultaneously [33]. Most preclinical studies showed that MSCs act through immunomodulation [1]. Another postulated mechanism is the induction of pro-fibrotic phenotypical changes in host fibroblasts, myofibroblasts and smooth muscle cells [34]. Nonetheless, definitive responses are not levels for elastin $* p<0.05$ versus Sham in analysis of variance with post hoc Student-Newman-Keuls analysis. d Summarized protein expression levels for Collagen I versus both PD-SVF and Sham in analysis of variance with post hoc Student-Newman-Keuls analysis. e Summarized protein expression levels for Collagen III $* * p<0.05$ versus both Sham and PD-SVF in analysis of variance with post hoc Student-Newman-Keuls analysis

yet available, and further studies focusing on the mechanisms of action are ongoing.

Our results provide novel evidence that autologous adipose SVF reduces collagen III in a rat model of chronic PD.

To date, 5 preclinical studies [18, 20,35-37], using the TGF- $\beta 1$ PD rat model, have evaluated the efficacy of locally 
TA ADSC injection. In two of our previous studies, we showed that in the acute PD phase, local injection of human ADSC (hADSC) or SVF prevents the collagen III and Elastin TA accumulation [18, 20]. In these studies, we injected the hADSC or autologous SVF 1 day after the TGF- $\beta 1$ treatment aiming to mimic the early phase of the disease $[18,20]$. Our results were in line with the two preclinical studies performed by Gokce et al $[36,37]$. Both these studies showed that TA injection of ADSCs was able to prevent rat TA fibrosis induced by TGF- $\beta 1$. Despite the promising results of these studies [20,36,37], it is known that only a small portion of PD patients present in the early phase of the disease. For this reason, we explored, in another our recent preclinical study, the efficacy of hADSC in a rat model of chronic PD [35]. In this study [35], we injected the hADSC 1 month from TGF- $\beta 1$ injection. 6 days following injection of TGF- $\beta 1$, the PD rat showed on histological analysis, less fibrosis than detected after 1 month in the previous study [22]. Furthermore, there was no corpora cavernosa fibrosis and erectile function impairment in the PD group compared with the sham rats. hADSC therapy was able to reduce the expression of TA collagen III but had no effect on collagen I and elastin expression [35].

In the current study, we aimed to assess the efficacy of SVF in the chronic phase of PD using the same animal model and the same study design of our previous experiment [35].

In chronic PD rat, no clear fibrotic plaques were detected on histological analysis. This results were confirmed by our previous study using the same animal model [35]. These data demonstrate that the fibrotic plaques in the TA tend to partially regress spontaneously after 60 days in the TGF- $\beta 1$ rat model of PD, which clearly is a limitation of the used animal model. Furthermore, in contrast to the previous study using the acute model of PD [22], we did not detect any significant corporal fibrosis. This may explain the lack of erectile function impairment in the PD group compared with the sham rats. These data were in contrast with the results of Gokce et al [36, 37]. In these studies, the PD animals showed erectile dysfunction and the presence of clear TA fibrotic plaque after 45 days the injection of TGF$\beta$. In the present study, we show that late SVF therapy is able to reduce the expression of collagen III but has no effect on the collagen I and elastin expression with a significant change in Collage I/III ratio. Type III collagen is a fibrillar collagen and it is secreted by fibroblasts and other mesenchymal cell types. It is secreted mainly during the wound healing process and it is one of the major players in several inflammation-related diseases such as lung injury, liver and renal fibrosis [4, 38, 39]. In the ECM, type III collagen constitutes the major part of the interstitial matrix together with type I collagen with a type I/III ratio of 2:1 [40]. During the early phase of wound healing, the type III collagen, act as a scaffold for fibroblast attachment. As consequence, initial granulation tissue contains mostly type III collagen and only a minor quantity of collagen I [41]. As wound healing progresses, this ratio is changed, leading to a type I/III ratio of $1: 2[2,4,40]$. The change in immature type III collagen may result in loss of tensile strength [40]. This shift is detected in many disorders such hypertrophic scars due to an amplified expression of type III pro-collagen mRNA [40]. Based on our study, SVF can restore the normal Collagen I/III ratio in an established TA fibrosis.

This study has inherent limitations including a lack of characterization of the injected cell types. However, others studies, using a similar isolation protocol, have examined the exact composition of SVF [9, 42-45]. More importantly, the evidence that the fibrotic plaques of TA tend to partially regress spontaneously after 60 days represents an important limit of the animal model which has been underreported in the literature.

\section{Conclusion}

Local injection of SVF in a rat model of chronic PD significantly decreased collagen III concentration in the TA. Further animal and clinical studies are needed to confirm the promising translational potential of this treatment strategy.

Acknowledgements We thank Arianna Bettiga, Fabio Benigni, Giorgia Colciago, Rita Van Bree, Catherien Luyten, Goedlieve Verbist, and Petra Steven for their technical contributions to the experiments in this study. This study was funded by the European Society for Sexual Medicine (ESSM) grant for basic medical research 2011 awarded to Fabio Castiglione and Maarten Albersen and by European Urological Scholarship Programme (EUSP) awarded to Fabio Castiglione Asif Muneer is supported by the NIHR Biomedical Research Centre UCLH.

\section{Compliance with ethical standards}

Conflict of interest The authors declare that they have no conflict of interest.

Publisher's note: Springer Nature remains neutral with regard to jurisdictional claims in published maps and institutional affiliations.

\section{References}

1. Milenkovic U, Albersen M, Castiglione F. The mechanisms and potential of stem cell therapy for penile fibrosis. Nat Rev Urol. 2019;16:79-97

2. Wynn Ta. Mechanism of fibrosis: therapeutic transplation for fibrotic disease. Nat Med. 2013;18:1028-40.

3. Vannella KM, Wynn TA. Mechanisms of organ injury and repair by macrophages. Annu Rev Physiol. 2017;79:593-617. 
4. Wynn TA. Cellular and molecular mechanisms of fibrosis. J Pathol. 2008;214:199-210.

5. Garaffa G, Trost LW, Serefoglu EC, Ralph D, Hellstrom WJ. Understanding the course of Peyronie's disease. Int J Clin Pr. 2013;67:781-8.

6. Abdel-Hamid IA, Anis T. Peyronie's disease: perspectives on therapeutic targets. Expert Opin Ther Targets. 2011;15:913-29.

7. Dellis A, Papatsoris A. Stem cell therapy for the treatment of Peyronie's disease. Expert Opin Biol Ther. 2017;17:407-13.

8. Goldring CE, Duffy PA, Benvenisty N, Andrews PW, Ben-David $\mathrm{U}$, Eakins R, et al. Assessing the safety of stem cell therapeutics. Cell Stem Cell. 2011;8:618-28.

9. Aronowitz JA, Lockhart RA, Hakakian CS. Mechanical versus enzymatic isolation of stromal vascular fraction cells from adipose tissue. + . 2015;4:713

10. Gentile P, De Angelis B, Pasin M, Cervelli G, Curcio CB, Floris $\mathrm{M}$, et al. Adipose-derived stromal vascular fraction cells and platelet-rich plasma: basic and clinical evaluation for cell-based therapies in patients with scars on the face. J Craniofac Surg. 2014;25:267-72.

11. Gumucio JP, Flood MD, Roche SM, Sugg KB, Momoh AO, Kosnik PE, et al. Stromal vascular stem cell treatment decreases muscle fibrosis following chronic rotator cuff tear. Int Orthop. 2016;40:759-64.

12. Han S, Sun HM, Hwang KC, Kim SW. Adipose-derived stromal vascular fraction cells: update on clinical utility and efficacy. Crit Rev Eukaryot Gene Expr. 2015;25:145-52.

13. Mazo M, Cemborain A, Gavira JJ, Abizanda G, Arana M, Casado $\mathrm{M}$, et al. Adipose stromal vascular fraction improves cardiac function in chronic myocardial infarction through differentiation and paracrine activity. Cell Transpl. 2012;21:1023-37.

14. Qiu X, Fandel TM, Ferretti L, Albersen M, Orabi H, Zhang H, et al. Both immediate and delayed intracavernous injection of autologous adipose-derived stromal vascular fraction enhances recovery of erectile function in a rat model of cavernous nerve injury. Eur Urol. 2012;62:720-7.

15. Haahr MK, Jensen CH, Toyserkani NM, Andersen DC, Damkier $\mathrm{P}$, Sorensen JA, et al. Safety and potential effect of a single intracavernous injection of autologous adipose-derived regenerative cells in patients with erectile dysfunction following radical prostatectomy: an open-label phase I clinical trial. EBioMedicine . 2016;5:204-10

16. Guillaume-Jugnot P, Daumas A, Magalon J, Jouve E, Nguyen PS, Truillet R, et al. Autologous adipose-derived stromal vascular fraction in patients with systemic sclerosis: 12-month follow-up. Rheumatol . 2016;55:301-6.

17. Bivalacqua TJ, Diner EK, Novak TE, Vohra Y, Sikka SC, Champion HC, et al. A rat model of Peyronie's disease associated with a decrease in erectile activity and an increase in inducible nitric oxide synthase protein expression. J Urol. 2000; 163:1992-8.

18. Castiglione F, Hedlund P, Weyne E, Hakim L, Montorsi F, Salonia $\mathrm{A}$, et al. Intratunical injection of stromal vascular fraction prevents fibrosis in a rat model of Peyronie's disease. BJU Int. 2018. https://doi.org/10.1111/bju.14570. [Epub ahead of print].

19. Albersen M, Kendirci M, Van der Aa F, Hellstrom WJ, Lue TF, Spees JL. Multipotent stromal cell therapy for cavernous nerve injury-induced erectile dysfunction. J Sex Med. 2012;9:385-403.

20. Castiglione F, Hedlund P, Van der Aa F, Bivalacqua TJ, Rigatti P, Van Poppel $\mathrm{H}$, et al. Intratunical injection of human adipose tissue-derived stem cells prevents fibrosis and is associated with improved erectile function in a rat model of Peyronie's disease. Eur Urol. 2013;63:551-60.

21. Castiglione F, Dewulf K, Hakim L, Weyne E, Montorsi F, Russo A, et al. Adipose-derived stem cells counteract urethral stricture formation in rats. Eur Urol. 2016;70:1032-41.
22. Castiglione F, Hedlund $\mathrm{P}$, Van der Aa F, Bivalacqua TJ, Rigatti P, Van Poppel $\mathrm{H}$, et al. Intratunical injection of human adipose tissue-derived stem cells prevents fibrosis and is associated with improved erectile function in a rat model of Peyronie's disease. Eur Urol. 2013;63:551-60.

23. Castiglione F, Bergamini A, Albersen M, Hannan JL, Bivalacqua TJ, Bettiga A, et al. Pelvic nerve injury negatively impacts female genital blood flow and induces vaginal fibrosis-implications for human nerve-sparing radical hysterectomy. Bjog. 2015;122:1457-65.

24. De la Peyronie F. Sur quelques obstacles quis'opposent à l'éjaculation naturelle de la semence. Mem Acad $R$ Chir. $1743 ; 1: 423-34$.

25. Sommer F, Schwarzer U, Wassmer G, Bloch W, Braun M, Klotz $\mathrm{T}$, et al. Epidemiology of Peyronie's disease. Int J Impot Res. 2002;14:379-83.

26. Gonzalez-Cadavid NF, Rajfer J. Mechanisms of disease: new insights into the cellular and molecular pathology of Peyronie's disease. Nat Clin Pr Urol. 2005;2:291-7.

27. Vernet D, Nolazco G, Cantini L, Magee TR, Qian A, Rajfer J, et al. Evidence that osteogenic progenitor cells in the human tunica albuginea may originate from stem cells: implications for peyronie disease. Biol Reprod. 2005;73:1199-210.

28. Gonzalez-Cadavid NF. Mechanisms of penile fibrosis. J Sex Med. 2009;6(Suppl 3):353-62.

29. Wynn TA, Ramalingam TR. Mechanisms of fibrosis: therapeutic translation for fibrotic disease. Nat Med. 2012;18:1028-40.

30. Caplan AI, Correa D. The MSC: an injury drugstore. Cell Stem Cell. 2011;9:11-5.

31. Caplan AI, Dennis JE. Mesenchymal stem cells as trophic mediators. J Cell Biochem. 2006;98:1076-84.

32. Yu FX, Su LF, Dai CL, Wang Y, Teng YY, Fu JH, et al. Inhibition of pancreatic stellate cell activity by adipose-derived stem cells. Hepatobiliary Pancreat Dis Int. 2015;14:215-21.

33. Caplan AI, Correa D. The MSC: an injury drugstore. Cell Stem Cell. 2011;9:11-5.

34. Sorrell JM, Caplan AI. Topical delivery of mesenchymal stem cells and their function in wounds. Stem Cell Res Ther. 2010 ;1:30.

35. Castiglione F, Hedlund P, Weyne E, Hakim L, Montorsi F, Bivalacqua $\mathrm{TJ}$, et al. Intratunical injection of human adipose tissue-derived stem cells restores collagen III/I ratio in a rat model of chronic Peyronie's disease. Sex Med. 2018;7:94-103. https:// doi.org/10.1016/j.esxm.2018.09.003.

36. Gokce A, Abd Elmageed ZY, Lasker GF, Bouljihad M, Braun SE, $\mathrm{Kim} \mathrm{H}$, et al. Intratunical injection of genetically modified adipose tissue-derived stem cells with human interferon alpha-2b for treatment of erectile dysfunction in a rat model of Tunica Albugineal fibrosis. J Sex Med. 2015;12:1533-44.

37. Gokce A, Abd Elmageed ZY, Lasker GF, Bouljihad M, Kim H, Trost LW, et al. Adipose tissue-derived stem cell therapy for prevention and treatment of erectile dysfunction in a rat model of Peyronie's disease. Andrology. 2014;2:244-51.

38. Wynn TA. Common and unique mechanisms regulate fibrosis in various fibroproliferative diseases. J Clin Invest. 2007; 117:524-9.

39. Duffield JS, Lupher M, Thannickal VJ, Wynn TA. Host responses in tissue repair and fibrosis. Annu Rev Pathol. 2013;8: 241-76.

40. El Sherif A, Yano F, Mittal S, Filipi CJ. Collagen metabolism and recurrent hiatal hernia: cause and effect? Hernia . 2006;10:511-20.

41. Wynn TA, Vannella KM. Macrophages in tissue repair, regeneration, and fibrosis. Immunity . 2016;44:450-62.

42. Lockhart RA, Aronowitz JA, Dos-Anjos Vilaboa S. Use of freshly isolated human adipose stromal cells for clinical applications. Aesthet Surg J. 2017;37(suppl_3):S4-s8. 
43. Aronowitz JA, Lockhart RA, Hakakian CS, Birnbaum ZE. Adipose stromal vascular fraction isolation: a head-to-head comparison of 4 cell separation systems \#2. Ann Plast Surg. 2016;77: 354-62.

44. Conde-Green A, Kotamarti VS, Sherman LS, Keith JD, Lee ES, Granick MS, et al. Shift toward mechanical isolation of adipose- derived stromal vascular fraction: review of upcoming techniques. Plast Reconstr Surg Glob Open. 2016;4:e1017.

45. Tremp M, Menzi N, Tchang L, di Summa PG, Schaefer DJ, Kalbermatten DF. Adipose-derived stromal cells from lipomas: isolation, characterisation and review of the literature. Pathobiology . 2016;83:258-66. 